\title{
Peculiarities of massive vector mesons and their zero mass limits
}

\section{To the memory of Raymond Stora}

\author{
Bert Schroer ${ }^{1,2, a}$ \\ ${ }^{1}$ CBPF, Rua Dr. Xavier Sigaud 150, Rio de Janeiro 22290-180, Brazil \\ 2 Institut für Theoretische Physik, FU-Berlin, Arnimallee 14, 14195 Berlin, Germany
}

Received: 9 April 2015 / Accepted: 29 May 2015 / Published online: 12 August 2015

(C) The Author(s) 2015. This article is published with open access at Springerlink.com

\begin{abstract}
Massive QED, in contrast with its massless counterpart, possesses two conserved charges; one is a screened (vanishing) Maxwell charge which is directly associated with the massive vector mesons through the identically conserved Maxwell current, while the presence of a particle-antiparticle counting charge depends on the matter. A somewhat peculiar situation arises for couplings of Hermitian matter fields to massive vector potentials; in that case the only current is the screened Maxwell current and the coupling disappears in the massless limit. In the case of self-interacting massive vector mesons the situation becomes even more peculiar in that the usually renormalizability guaranteeing validity of the first order power-counting criterion breaks down in second order and requires the compensatory presence of additional Hermitian $H$-fields. Some aspect of these observation have already been noticed in the BRST gauge theoretic formulation, but here we use a new setting based on string-local vector mesons which is required by Hilbert space positivity ("off-shell unitarity"). This new formulation explains why spontaneous symmetry breaking cannot occur in the presence of higher spin $s \geq 1$ fields. The coupling to $H$-fields induces Mexican hat-like self-interactions; they are not imposed and bear no relation with spontaneous symmetry breaking; they are rather consequences of the foundational causal localization properties realized in a Hilbert space setting. In the case of self-interacting massive vector mesons their presence is required in order to maintain the first order power-counting restriction of renormalizability also in second order. The presentation of the new Hilbert space setting for vector mesons which replaces gauge theory and extends on-shell unitarity to its off-shell counterpart is the main motivation for this work. The new Hilbert space setting also shows that the second order Lie-algebra structure of self-interacting vector mesons is a consequence of the principles of QFT and promises a deeper understanding of the origin of confinement.
\end{abstract}

\footnotetext{
a e-mail: schroer@zedat.fu-berlin.de
}

\section{Introduction}

The theoretical interest in massive vector mesons can be traced back to Schwinger's conjecture [1], which states that "massive QED" leads to "charge screening". The analogy to the quantum mechanical theory of superconductivity, where the long-range vector potentials of electromagnetism become short ranged, lends plausibility to Schwinger's quantum field theoretical conjecture. This idea was made precise in a theorem by Swieca [2,3], who showed that the Maxwell current of a massive vector meson, independent of whether it interacts with complex or Hermitian matter fields (in the sequel referred to a $H$-matter), always leads to a vanishing charge of the identically conserved Maxwell current.

The proof uses analytic properties of matrix elements (form factors) of identically conserved currents associated with a field strength tensor of a massive vector meson. We will refer to this phenomenon as "Schwinger-Swieca screening". The global particle-antiparticle number conservation remains unaffected by the vanishing of the Maxwell charge. In the case of a coupling to $H$-matter (the abelian Higgs model) there is only the screened Maxwell charge.

Massive QED does not require the presence of $H$-particles and massive vector mesons do not owe their mass to spontaneous symmetry breaking (SSB). As will be shown in the present work, the mass of vector mesons bears no intrinsic (physically meaningful) relation with a spontaneous symmetry breaking "Higgs mechanism". As in the case of quantum mechanical superconductivity, where long-range vector potentials become short ranged without adding new degrees of freedom, massive QED does not require the presence of additional $H$-matter.

The situation changes in the presence of self-couplings between massive vector mesons as will be explained in the sequel. A necessary restriction on first order couplings from the requirement of renormalizability is the power-counting restriction for the short distance scale dimension of the first 
order interaction density $d_{\mathrm{sd}}^{\text {int }} \leq 4$. In all known renormalizable models, except those involving self-interacting massive vector mesons, this bound secures the preservation of renormalizability to all orders. This "golden rule" of renormalization theory is satisfied in first order self-interactions between massive vector mesons, but is lost in second order; although there exists a unique first order gauge invariant density for massive self-interacting vector mesons with $d_{\mathrm{sd}}^{\text {int }}=4$, the implementation of second order gauge invariance for the $S$-matrix induces terms which violate the power-counting restriction.

It turns out that this second order violation can only be prevented by a compensation in which an additional field couples to the vector mesons in compliance with the first order power-counting restriction but a violation in second order. This coupling must be such that the contributions from both second order terms cancel. The charge neutrality of vector mesons requires the new fields to be Hermitian; it turns out that the compensation can only be achieved with scalar $H$-fields. Such a cancelation is reminiscent of short-distance improvements from compensations between different spin components in supersymmetric interactions, but it is well known that they did not suffice in order to remain within the power-counting restriction.

However, for the case at hand the compensation works; $A_{\mu}$ self-couplings and $A-H$ coupling collaborate in such a way that renormalizability is preserved in second order. This scenario was first elaborated in the setting of BRST gauge theory by Scharf [4] and Collaborators [5], for a more recent account see also [6]. They start from the perturbative formal representation of the $S$-matrix ${ }^{1}$ in terms of the adiabatic limit of time-ordered products of the first order interaction density and impose BRST gauge invariance in the form $\mathfrak{s} S=0$, where $\mathfrak{s}$ denotes the nilpotent BRST $s$-operation.

This turns out to be very restrictive; in the case of only one $H$ field all couplings are determined. As expected, in the limit of zero mass vector mesons the $H$ decouples from the $A_{\mu}$ and becomes a free field, whereas the massless selfinteracting vector mesons take the form of a (massless) YangMills (YM) interaction.

Besides the BRST gauge formalism, needed for the definition of the gauge invariant $S$-matrix, no other property has been used to derive this result. In particular there is no reference to SSB which starts with the Mexican hat potential, whereas in the present setting it is induced from the imposition of second order gauge invariance.

What is meant by "induction" is best explained in the simpler abelian Higgs model. The starting point of any perturbation theory is a set of interaction-defining free fields and a first order coupling strength; in the present case these are three

\footnotetext{
1 The physical $S$-matrix is the adiabatic limit of the Bogoliubov generating operator $S$-functional.
}

parameters, namely the masses of the vector meson and the $H$, and the first order coupling strength in $g A_{\mu} A^{\mu} H$. Among the terms which the imposition of gauge invariance (BRST $\mathfrak{s}$-invariance) on the $S$-matrix induces in second order one finds the tri- and quadri-linear terms, which can be written in the form of a Mexican hat potential which only depends on $g$ and the ratios of the two masses [4]. This kind of induction, which generates new interactions without enlarging the number of coupling parameters, is a consequence of the gauge invariance of $S$.

The construction of the (abelian) Higgs model in terms of a SSB prescription is very different. It starts with the Lagrangian of two-parametric scalar QED and breaks gauge invariance in terms of a shift in field space. A subsequent gauge transformation converts the resulting expression, apart from the unphysical parametrization, into the same expression as that obtained by imposing gauge invariance. An easy test, which shows that the shift prescription has no relation to a physical SSB, is to pass to the limit of a vanishing shift parameter. In a genuine SSB the model would return to its unbroken form which was 2-parametric scalar QED. This does not happen, the physically meaningless application of a gauge transformation after breaking of gauge invariance prevents this return.

A more profound argument results from the observation that the Maxwell current of the Higgs model, as any Maxwell current of a massive vector meson, leads to a screened charge $Q=0$, whereas the conserved current of a genuine SSB model implies $Q=\infty$. A conserved current whose "would be" symmetry-generating charge diverges is actually the definition of a SSB, whereas the shift in field space is a device which prepares such a current.

It is well known that such situations can be generated from quartic interactions between scalar particles which are invariant under the action of compact groups. The shift in field space is a convenient tool to construct a first order interaction whose renormalized perturbation theory preserves current conservation but leads to the divergence of some of the charges. It does not create masses but rather prepares the kind of special interaction between massive and massless ("Goldstone bosons") free fields which maintains current conservation but causes the long-distance divergence of some charges.

This construction is limited to interactions to interactions involving low spins $s<1$. The coupling of vector mesons to scalar fields prevent the latter from causing a SSB. The usual argument is that the scalar particles become gauge-dependent and local gauge invariance is not really a physical symmetry which can be broken. This argument involves formal steps with unclear physical content; gauge "symmetries" are not physical symmetries and it is not clear what one is breaking.

A less formal more physical argument is to look directly at the gauge invariant conserved current which the coupling to the vector meson has transformed into an identi- 
cally conserved Maxwell current. The charge of Maxwell currents of massive vector mesons vanish as a consequence of the Schwinger-Swieca charge screening; this is a gauge-invariant phenomenon (it involves only gaugeinvariant observables). A screened charge $Q=0$ is very different from a SSB charge $Q=\infty$; between the two is the nontrivial charge $Q<\infty$, which is the generator of a symmetry. As soon as higher spin fields couple to scalar fields, the latter are prevented to undergo SSB; the scalar particles have to follow the more restrictive nature of higher spin interactions places the vector mesons into the driver's seat. In this way those scalar fields which couple to $s=1$ fields are prevented from transforming into their SSB mode.

In the string-local field (SLF) Hilbert spaces setting, whose presentation is the main purpose of the present paper, the interaction with string-local vector mesons converts the scalar fields into interacting string-local fields. In this setting there is simply no gauge symmetry which could undergo spontaneous breaking; rather all point-local fields which couple to string-local vector potentials lose their point-local nature and become string-local. Such fields are not local observables. But in contrast to their gauge-theoretic counterparts they are physical, since they act in a Hilbert space.

The Higgs mechanism leads to the correct coupling of $H$ with massive vector mesons but it fails to reveal the raison d'etre of the $H$-field which has nothing to do with symmetry breaking and mass generation. The vector mesons in massive QED do not need the presence of a mass-generating $H$, it is a renormalizable theory by itself. As pointed out before, the situation changes radically in the presence of self-interacting massive vector mesons. In that case the theoretical reason for its existence is the second order preservation of renormalization. As a result of the connection between renormalizability and causal localizability, the $H$ plays a fundamental role for the consistency of the $W^{ \pm}, Z$ interactions in the Standard Model.

The preservation of second order reormalizability with the help of an $H$-particle is vaguely reminiscent of the introduction of vector mesons to convert the first order nonrenormalizable 4-Fermi interaction into a renormalizable coupling, except that a second order compensation mechanism is much more sophisticated. The fundamental nature of the LHC discovery is in no way affected by this new role assigned to the $H$, but in particle theory it is important to distinguish between prescriptions for construction of models and their intrinsic properties.

It is an interesting question why this was not seen in the standard BRST formulation in terms of Feynman rules. The answer is that in an off-shell gauge formalism one usually presents the perturbative rules, but one does not focus on the explicit construction of a gauge-invariant on-shell unitary $S$ matrix. In $[4,5]$ the BRST formalism was especially adjusted to that problem by what the authors called the "causal gauge invariance" (CGI) setting which is based on the EpsteinGlaser operator formulation. This leads to a rather clear distinction between quasiclassical pictures of symmetry breaking and gauge-induced $H$-self-interactions.

This CGI setting permits a direct perturbative construction of a unitary gauge-invariant $S$-matrix for a $g A \cdot A H$ coupling of a massive vector meson with a Hermitian field; in this way it highlights the second order induction mechanism which leads to the Mexican hat $H$ self-interaction. It bears no relation with generating masses of vector mesons; whereas massive QED does not need the presence of $H$ fields in order to generate the mass of the vector meson, their presence is necessary in all interaction involving self-interacting massive vector mesons and the CGI formulation reveals the correct reasons.

Interactions in QFT cannot generate masses in any material sense; masses of interacting-defining elementary fields (i.e. those in terms of which the first order interaction is defined) must be put in, and higher order renormalization theory preserves them. What one expects from the theory is that the mass of possible bound states, which correspond to composites of the elementary fields, can be computed within the theory; but this does not seem to be possible within a perturbative setting.

The main concern in the present work is to replace the "ghostly" gauge theory by a new ghost-free Hilbert space formulation of interactions for $s=1$ fields.

Gauge theory is not a substitute of a Hilbert space formulation, but it is a rather successful placeholder. The awareness about its makeshift status was much stronger in the past than it is now. There were several valiant attempts to avoid the use of indefinite metric by Mandelstam [7] and also DeWitt [8]; their failure also revealed that a description which is consistent with positivity ("off-shell unitarity") requires major new conceptual investments beyond what was known at that time.

As a result of impressive observational successes of gauge theory in its applications to the Standard Model, this problem moved gradually into the background. Physicists of the older generation sometimes express their surprise that the conceptual incomplete gauge theory works much better than expected; occasionally they think of this success in terms of as a results of unmerited luck (I thank Raymond Stora for sharing his views on this problem).

The new Hilbert space setting reveals what can be correctly described within the perturbative gauge theory setting and what problems remain outside its physical range. The vacuum sector, generated by the application of gauge invariant observables to the vacuum state, and the gauge-invariant $S$-matrix in the presence of a mass gap are within its physical range, whereas the construction of causally localized fields 
and their asymptotically related particles states remain outside.

This is a serious limitation in particular in zero mass limits when the construction of the gauge-invariant $S$-matrix fails and one has to take recourse to calculational recipes without understanding their connection with the foundational spacetime localization properties. Such problems related to the long-distance behavior of fields (infrared problems in momentum space) are outside the range of gauge theory.

Local gauge symmetry is a well-defined concept in classical field theory, but it clashes with the Hilbert space positivity of quantum theory. Instead of representing a physical symmetry, it is a formal device for extracting a physical subtheory. This is a consequence of the fact that locality in a Krein space, in contrast to Einstein causality in Hilbert space, has only a formal but no physical motivation. Its become particularly annoying in the massless limit, when scattering amplitudes suffer from infrared divergencies and one is forced to describe collisions in terms of momentum-space prescriptions instead of spacetime localization properties of physical fields. Such problems can only be solved in a Hilbert space setting; the operators in QFT which corresponds to long-range (Coulomb) potentials in quantum mechanics are string-local fields [9].

The new SLF theory should explain why self-interacting vector mesons lead to a Lie-algebra structure with only one coupling strength. In the BRST gauge setting this arises as a consistency condition of the formalism [4]. This is not surprising, since the BRST formalism is the result of an adaptation of classical gauge theory to the exigencies of QFT. ${ }^{2}$ The physically more relevant question is whether the Lie-algebra structure can be derived solely from the causal localization principles of QFT in a Hilbert space setting without referring to the classical mathematics of fiber bundles. In Sect. 6 it will be shown that this is indeed the case.

A somewhat surprising result is that the use of covariant string-local potentials permits a simple and amusing description of topological effects which are present in the zero mass limit, as the breakdown of Haag duality and the closely related Aharonov-Bohm effect (Sect. 3).

The interest in string-local fields, as they are used in the present work, started with the solution of an old problem which goes back to Wigner. In his famous 1939 paper, which contains the classification of all positive energy representations of the Poincaré group, Wigner found besides the massive and the zero mass finite helicity representation a third massless representation class which he referred to as "infinite spin". Whereas there was no problem to associate point-local covariant fields with the first two classes, the problem of a field theoretic description of the third class remained for a long time open. In [10] it was shown that this class cannot

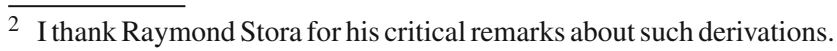

be described in terms of point-local Wightman fields. Using methods of modular localization ${ }^{3}$ Brunetti et al. [11] showed that these representations permit causal localization in arbitrary narrow space-like cone regions in Minkowski space (for historical remarks on modular localization see $[12,13])$.

This suggested that it should be possible to associate covariant fields localized on space-like semi-infinite strings (the "core" of arbitrary narrow space-like cones) with such a situation; such fields were then explicitly constructed in [14]. In that work arguments were given which suggested that such models do not admit composite point-local fields; they were significantly extended in forthcoming work by $\mathrm{Ch}$. Köhler. An elegant proof of their absence was recently given in [15].

In [14] also string-local fields for the massive and zero mass finite helicity Wigner representations were constructed in the hope that they could be useful in perturbation theory and may lead to a ghost-free Hilbert space formulation of $s=1$ interactions which replaces gauge theory. These ideas were further pursued in [12,16-20]. The present work is a continuation of these ideas in the context of explicit second order calculations in the presence of string-local massive vector potentials. The presentation of the general formalism in the presence of string-crossings will be contained a forthcoming paper by Jens Mund; further details as regards applications to interacting string-local fields will be addressed in joint work by Mund and the present author.

It had been known for a long time $[21,22]$ that within the setting of algebraic QFT the field-particle relation in the presence of a mass gap can always be described in terms of operators localized in arbitrary narrow space-like cones (whose cores are semi-infinite space-like strings). It is interesting to note that the ideas which led to these results arose from a previous publication of the authors in which they removed a remaining loophole in the proof of Swieca's screening theorem [23].

The approach presented in this paper may be seen as an adaptation of those structural results to the requirements of renormalized perturbation theory in terms of string-local fields.

This work is organized as follows.

The next section presents conceptual aspects of the Hilbert space setting and compares them with the formalism of operator gauge theory.

The third section contains a simple but somewhat surprising application of free string-local fields.

The problem of their role in interactions is taken up in Sect. 4.

Section 5 presents second order perturbative results which includes in particular the interaction of massive vector mesons with Hermitian matter (the Higgs model).

\footnotetext{
3 Modular localization is an intrinsic formulation of causal localization which does not rely on the use of particular field "coordinatizations".
} 
In Sect. 6 it is shown that the Lie-algebra structure of self-interacting vector mesons is a consequence of causal localization in Hilbert space.

The concluding remarks present an outlook about what can be expected from this new setting concerning unsolved problems of infrared divergencies such as "infraparticles" in QED and confinement in QCD.

\section{Formal analogies and conceptual differences between CGI and SSB}

For the convenience of the reader we start with a compilation of formulas as they are used in the CGI formulation of BRST gauge theory for the construction of the gauge-invariant $S$ matrix [4].

$$
\begin{aligned}
& \mathfrak{s} A_{\mu}^{K}=\partial_{\mu} u^{K}, \mathfrak{s} \phi^{K}=u^{K}, \mathfrak{s} \hat{u}^{K}=-\left(\partial A^{K}+m^{2} \phi^{K}\right) \\
& \mathfrak{s} B:=i[Q, B], Q=\int \mathrm{d}^{3} x\left(\partial^{v} A_{\nu}^{K}+m^{2} \phi\right) \overleftrightarrow{\partial}{ }_{0} u .
\end{aligned}
$$

Here the superscript $K$ refers to the Krein space in which these operators are realized, $Q$ is the so-called ghost charge, whose properties ensure the nilpotency $\left(\mathfrak{s}^{2}=0\right)$ of the BRS $\mathfrak{s}$-operation. The $A_{\mu}^{K}$ is a massive vector meson in the Feynman gauge and $\phi$ is a free scalar field of the same mass but with a two-point function of opposite sign (a kind of negative metric Stückelberg field); these two fields carry the indefinite metric which requires to replace the Hilbert space by a Krein space (iteratively created by iterative application of the operators to the vacuum). The "ghosts" $u, \hat{u}$ are free "scalar fermions" whose presence is necessary in order to recover the vacuum sector of the local observables acting in a Hilbert space and the unitary $S$-matrix in the form of $\mathfrak{s}$-invariant operators.

These rules in terms of free fields suffices for the construction of the gauge-invariant $S$-matrix; the extension to (gauge-variant) interacting fields follows similar formal rules as those for interacting fields with lower spin $s<1$. The $Q$-charge and the $\mathfrak{s}$ participate in the perturbation theory of interacting fields.

The BRST formalism is a pure perturbative tool; structural properties (TCP, spin and statistics,...) as well as the physical causal locality properties of which they are consequences require the Hilbert space positivity. The tools one needs for non-perturbative constructions and the derivation of structural theorems (Schwartz inequality,...) are not available in a Krein space setting. Quantum gauge theory is limited to the combinatorial manipulations of perturbation theory.

It should not come as a surprise that the ghost formalism (the $\mathfrak{s - c o h o m o l o g y ) , ~ u n l i k e ~ t h e ~ l a t e r ~ S L F ~ H i l b e r t ~ s p a c e ~ f o r - ~}$ mulation (differential forms on the $d=1+2$ unit de Sitter space of space-like directions), has no relation with space- time. The BRST rules were not derived from localization principles of QFT but they were found in the course of trying to recover unitarity of the $S$-matrix ("on-shell unitarity") in a Krein space setting. In order to arrive at the BRST operational formulation it needed several improvements of the original unitarity arguments of 't Hooft-Veltman (FaddeevPoppov, Slavnov) in order to reach the formally elegant ghost formalism of Becchi-Rouet-Stora-Tyutin.

Although the use of these prescriptions turned out to be essential for the success of the Standard Model, their conceptual relation with the foundational principles remained unclear. QFT is the realization of causal localization in a Hilbert space; without positivity ("unitarity") there is no probability interpretation and hence the relation with quantum theories foundational property is lost in gauge theory; it can only be recovered in special (gauge-invariant) situations. It needs to be emphasized that classical gauge theory is not affected by these shortcomings since the foundational Hilbert space structure is characteristic of quantum theory. Hence it is not surprising that the SLF Hilbert space description is outside Lagrangian quantization (but not outside perturbation theory).

As already mentioned in the introduction, massless $s \geq$ 1 covariant tensor potentials are necessarily string-local. Massive point-like potentials exist, but as a result of their short-distance dimension $d_{\mathrm{sd}}^{s}$ (point) $=s+1$ their interactions are nonrenormalizable since interactions formed with them violate the power-counting limit $d_{\mathrm{sd}}^{\text {int }} \leq 4$. The fact that the smallest possible short-distance dimension of string-local fields is $d_{\mathrm{sd}}^{s}$ (string) $=1$ suggests that there may be renormalizable string-local interactions. But the power-counting limit is not the only restriction, physics demands the existence of sufficiently many local observables generated by pointlocal fields and the preservation of string-localization. Furthermore the $S$-matrix in models with a mass gap should be independent of the string directions $e$; although fields may be string-local, the particles which they interpolate remain those string-independent objects whose wave-function spaces were classified by Wigner. In the following we will show how these requirements can be met for massive vector mesons.

We start from a massive vector potential (the Proca field) and define its associated string-local potential in terms of the Proca field strength

$$
\begin{aligned}
F_{\mu \nu}(x) & =\partial_{\mu} A_{\nu}^{P}(x)-\partial_{\nu} A_{\mu}^{P}(x), \\
A_{\mu}(x, e) & =\int_{0}^{\infty} F_{\mu \nu}(x+\lambda e) e^{\nu} \mathrm{d} \lambda, \\
\phi(x, e) & =\int_{0}^{\infty} A_{\mu}^{P}(x+\lambda e) e^{\mu} \mathrm{d} \lambda, \quad e^{2}=-1 .
\end{aligned}
$$

Whereas the short-distance dimension of the Proca potential and its field strength is $d_{\mathrm{sd}}^{P}=2$, the string-local vector potential and its scalar "escort" $\phi$ have $d_{\text {sd }}=1$. We could of 
course change the "population density" of degrees of freedom on the semi-infinite line from $p(\lambda) \equiv 1$ (as above) to any other smooth function which approaches 1 asymptotically without leaving the local equivalence (Borchers) class; but if we want in addition to uphold a linear relation, which follows from (2),

$$
A_{\mu}(x, e)=A_{\mu}^{P}(x)+\partial_{\mu} \phi(x, e),
$$

we must use the same $p(\lambda)$. Formally this corresponds to the possibility of gauge changes in $(1) ; p(\lambda)$ changes preserve relative localization and a fortiori do not change the particle content in the presence of interactions.

In contrast to the "virtual" strings of anyons/plektons [24], which, similar to cuts in complex function theory, may be displaced as long as crossings are prevented, the strings of string-local fields are "real". Needless to mention that the string-local potentials are the only vector potentials which permit a $m \rightarrow 0$ limit.

For the following it turns out to be convenient to express (2) as linear relations between $v$-intertwiners

$$
\begin{aligned}
& A_{\mu}^{P}(x)=\frac{1}{(2 \pi)^{3 / 2}} \int\left(e^{i p x} \sum_{s_{3}=-1,0,1} v(p)_{\mu, s_{3}} a^{*}\left(p, s_{3}\right)+h . c .\right) \\
& v_{\mu, s_{3}}^{A}(p, e)=v_{\mu, s_{3}}(p)+i p_{\mu} v_{s_{3}}^{\phi}, v_{s_{3}}^{\phi}(p)=\frac{i v_{s_{3}} \cdot e}{p \cdot e+i \varepsilon}
\end{aligned}
$$

where the second line is the relation (3) rewritten as a linear relation between the three intertwiners. Here $v$ refers to the intertwiner between the 3-component unitary Wigner representation and the covariant vector-components of the Proca potential. Using the differential form calculus on the $d=1+2$ de Sitter space of space-like directions, we define exact 1 - and 2 -forms,

$u=d_{e} \phi=\partial_{e_{\alpha}} \phi d e^{\alpha}, v_{s_{3}}^{u}=i\left(\frac{v_{\alpha, s_{3}}}{p \cdot e}-\frac{v \cdot e}{(p \cdot e)^{2}} p_{\alpha}\right) d e^{\alpha}$,

$\hat{u}=d_{e}\left(A_{a} d e^{\alpha}\right)=\int_{0}^{\infty} \mathrm{d} \lambda F_{\alpha \beta}(x+\lambda e) d e^{\alpha} \wedge d e^{\beta}$,

$v^{\hat{u}}=p_{\alpha} \frac{v_{\beta, s_{3}}}{p \cdot e} d e^{\alpha} \wedge d e^{\beta}$.

They are field-valued differential forms with $d_{\mathrm{sd}}=$ 1 which in a certain sense represent the Hilbert space counterpart of the cohomological BRST ghost formalism in Krein space. To maintain the simplicity of the covariant formalism, the differential forms on the unit $d=1+2$ de Sitter space are viewed as restrictions of the four-dimensional directional $e$-formalism. The notation $u, \hat{u}$ suggests that they may play the role of the differential form analog of the ghost in the BRST formalism.

These field-valued differential forms are natural extensions of string-local fields; together with $A_{\mu}(x, e)$ and $\phi(x, e)$ they are the only covariant string-local members of the linear part of the local equivalence class of the free Proca field with $d_{\mathrm{sd}}=1$. Their ghost counterparts are important in the off-shell BRST formalism, but (apart from the appearance of $u^{(K)}$-terms in the $Q_{\mu}$ formalism) they disappear in the formula for $\mathrm{S}$.

The 2-point Wightman functions of string-local objects can be calculated from their intertwiners or directly in terms of the line integral representation of the string-local $A, \phi$ fields. One obtains ${ }^{4}$

$$
\begin{aligned}
\left\langle A_{\mu}(x, e) A_{v}\left(x^{\prime}, e^{\prime}\right)\right\rangle= & \frac{1}{(2 \pi)^{3}} \int e^{-i\left(x-x^{\prime}\right) p} M_{\mu v}^{A}(p) \frac{\mathrm{d}^{3} p}{2 p_{0}} \\
M_{\mu \mu^{\prime}}^{A}\left(p ; e, e^{\prime}\right)= & -g_{\mu \mu^{\prime}}-\frac{p_{\mu} p_{\mu^{\prime}}\left(e \cdot e^{\prime}\right)}{(p \cdot e-i \varepsilon)\left(p \cdot e^{\prime}+i \varepsilon\right)} \\
& +\frac{p_{\mu} e_{\mu^{\prime}}}{(p \cdot e-i \varepsilon)}+\frac{p_{\mu} e_{\mu^{\prime}}^{\prime}}{\left(p \cdot e^{\prime}+i \varepsilon\right)}
\end{aligned}
$$

and similar expressions for $M^{\phi}$ and mixed vacuum expectations $M_{\mu}^{A, \phi}$. The occurrence of the latter (which vanish in the gauge setting) is the price to pay for maintaining off-shell positivity. Only point-like (generally composite) local observables and the $S$-matrix are independent of $e$; but their perturbative computation requires the use of stringlocal fields. The $p \cdot e \pm i \varepsilon$ terms in the denominator are the momentum-space expressions which correspond to the line integrals in the creation, respectively, annihilation components.

The time-ordered propagators are formally obtained in terms of the substitution

$\frac{\mathrm{d}^{3} p}{2 p_{0}} \rightarrow \frac{1}{2 \pi} \frac{1}{p^{2}-m^{2}+i \varepsilon} \mathrm{d}^{4} p$,

together with the Epstein-Glaser minimal scaling rule which allows the appearance of undetermined counterterms in the case the scale dimension of the propagator is $d \geq 4$. For later use (Sect. 3) we also note

$$
\begin{aligned}
& \partial^{\mu} \int e^{-i \xi \cdot p} \frac{p_{\mu} p_{v}}{\left(p^{2}-m^{2}+i \varepsilon\right)(p \cdot \varepsilon+i \varepsilon)} \\
& \quad=\partial^{v} \int_{0}^{\infty} \delta(\xi+s e) d s=: \partial^{v} \delta_{e}(\xi) .
\end{aligned}
$$

Whereas in the SLF Hilbert space formalism the particle creation and annihilation operators are directly associated to Wigner-particle states, the Krein space substitute of the Proca potential

$A_{\mu}^{P, K}:=A_{\mu}^{K}-\partial_{\mu} \phi^{K}, \quad \mathfrak{s} A_{\mu}^{P, K}=0$

\footnotetext{
4 More details will be contained in forthcoming work by Mund.
} 
only "emulates" such states inside matrix elements of gaugeinvariant operators. For the $S$-matrix one has

$$
\left\langle q_{1}^{K} . . q_{m}^{K}\left|S^{K}\right| p_{1}^{K}, . . p_{n}^{K}\right\rangle=\left\langle q_{1} . . q_{m}|S| p_{1}, . . p_{n}\right\rangle
$$

where the Krein space vector meson states are obtained by successive application of $A_{\mu}^{P . K}$ to the vacuum state. The gauge formalism can generally not prevent "leakage" of physical states defined by $\mathfrak{s}|\psi\rangle=0$ into unphysical regions of Krein space.

Besides the local observables, the only known shared global operator is the $S$-matrix. A considerable conceptual difference is that in SLF in addition to the perturbative definition of $S$ there also exists a non-perturbative derivation in terms of large time asymptotic properties of interpolating fields in Hilbert space (on-shell unitarity from a massshell restriction of off-shell unitarity). In that case the $e$ independence of the $S$-matrix is a result of the extension of scattering theory to space-like cone localized operators (strings as limits of space-like cones) [22].

In both perturbative settings the perturbative calculations impose the requirement of $\mathfrak{s}$ or $d_{e}$ invariance on the formal time-ordered expression of the Bogoliubov formalism. In the SLF setting one expects that the perturbative $S$-matrix formalism can be extended to string-local physical fields whose perturbative correlation functions are independent of the e-directions of internal propagators. Such a distinction between the string dependence of interacting fields, whose vacuum expectation values one wants to calculate, and $e^{\prime} s$, which appear in internal propagators of their perturbative expansion, has no counterpart in gauge theory. Despite some formal analogies, the conceptual differences between the global BRST cohomology of the $\mathfrak{s}$ and the geometric $d$ differential form calculus acting on the spacetime string directions remain formidable.

\section{The breakdown of Haag duality and a new look at the Aharonov-Bohm effect}

An example of an effect which cannot be described in the Krein space setting of point-like vector potentials, but that is correctly accounted for in the string-local Hilbert space formalism, is the breakdown of Haag duality and the closely related Aharonov-Bohm effect. It is instructive to illustrate this in some detail in terms of Wilson loops.

For the following calculations it turns out to be convenient to work with regularized electric and magnetic field strengths. Let $B$ be a small ball centered at the origin and $\rho, \sigma$ functions with supp $\rho \subset B$. Then we define regularized field strengths in terms of convolutions with $\rho$
$\vec{E}_{\rho}(\vec{x})=\int \vec{E}\left(\vec{x}-\vec{x}^{\prime}\right) \rho\left(\vec{x}^{\prime}\right) \mathrm{d}^{3} \vec{x}^{\prime}, \quad \vec{H}_{\sigma}(\vec{x})=\cdots$

The corresponding regularized fluxes through a surface $D$ are

$E_{\rho}(D)=\int_{D} \vec{E}_{\rho}(\vec{x}) \mathrm{d} \vec{D}, \quad H_{\sigma}(D)=\int_{D} \vec{H}_{\sigma}(\vec{x}) \mathrm{d} \vec{D}$

Using the equal time commutation relation between the electric and magnetic field strengths, one obtains for regularized electric and magnetic surface fluxes through $D$, respectively, $\hat{D}$

$$
\begin{aligned}
& 4 \pi i\left[E_{\rho}(D), H_{\sigma}(\hat{D})\right]=\int \vec{g}_{D, \rho}(\vec{x}) \operatorname{curl} \vec{g}_{\hat{D}, \sigma}(\vec{x}) \mathrm{d}^{3} \vec{x} \\
& \vec{g}_{D, \rho}(\vec{x})=\int \vec{g}_{D}\left(\vec{x}-\vec{x}^{\prime}\right) \rho\left(\vec{x}^{\prime}\right), \quad \vec{g}_{D}(\vec{f}) \\
& \quad=\int_{D} \vec{f} \mathrm{~d} \vec{D} ; \vec{g}_{\hat{D}, \sigma}(x)=\ldots,
\end{aligned}
$$

where the vector-valued functions $\vec{g}_{D, \rho}(\vec{x}), \vec{g}_{\hat{D}, \sigma}(\vec{x})$ are obtained by regularizing the vector-valued surface distributions $\vec{g}_{D}(\vec{x})$ as define in the second line. Since the divergence of $\vec{E}_{\rho}(\vec{x})$ and $\vec{H}_{\sigma}(\vec{x})$ vanishes, the corresponding flux $H_{\rho}(\hat{D})$ depends only on $\partial \hat{D}$; hence it can be localized on any surface spanning $\partial \hat{D}$.

Thinking in pure geometric terms, one would expect that the fluxes are localized on the tori $\mathcal{T}=\partial \mathcal{D}+\mathcal{B}, \hat{\mathcal{T}}=\partial \hat{D}+$ $B$, so that in the case they interpenetrate but do not touch the commutator vanishes. But an explicit calculation for such a situation shows that this is not true.

Taking for $D$ and $\hat{D}$ the discs

$D=\left\{\vec{x} \in \mathbb{R}^{3} ; x_{3}=0, x_{1}^{2}+x_{2}^{2}=1\right\}$,

$\hat{D}=\left\{\vec{x} \in \mathbb{R}^{3} ; x_{1}=0,\left(x_{2}-1\right)^{2}+x_{3}^{2}=1\right\}$,

whose associated interpenetrating tori $\mathcal{T}=\partial D+B, \hat{T}=$ $\partial \hat{D}+B$ do not intersect for a sufficiently small $B$, one finds

$4 \pi i\left[E_{\rho}(D), H_{\sigma}(\hat{D})\right]=\int \rho(\vec{x}) \mathrm{d}^{3} x \cdot \int \sigma(\vec{x}) \mathrm{d}^{3} x$

This result for this straightforward but somewhat lengthy calculation has been taken from old (unfortunately unpublished) manuscript by Leyland et al. [25]. It is what one expects from the picture of a magnetic flux through a torus which passes through the regularized electric surface. It is independent of the electric and magnetic surfaces $D$ and $\hat{D}$ as long as one does not change their boundaries.

The purpose of their calculation was to show the breakdown of Haag duality for the system of localized operator algebras generated by a free field QED field strength $F_{\mu \nu}(x)$. The terminology will be explained in the sequel. 
Causal localization properties of QFT are best described in the setting of algebraic QFT in which models are defined in terms of their net of algebras of causally localized observables. Denoting such algebras localized in the compact spacetime region $\mathcal{O}$ as $\mathcal{A}(\mathcal{O})$, the causality properties of QFT are expressed in terms of two independent relations,

$$
\mathcal{A}(\mathcal{O})=\mathcal{A}\left(\mathcal{O}^{\prime \prime}\right), \quad \mathcal{A}(\mathcal{O}) \subseteq \mathcal{A}\left(\mathcal{O}^{\prime}\right)^{\prime} .
$$

Here the first equation is the causal completeness property (the causal completion $\mathcal{O}^{\prime \prime}$ is the two times applied causal complement $\mathcal{O} \rightarrow \mathcal{O}^{\prime}$ ) and the second relation (in which the dash on the algebra denotes its commutant) is the algebraic formulation of Einstein causality.

The causal completeness property is a physically indispensable part of causality, although it often does not receive the same attention as Einstein causality. ${ }^{5}$ It corresponds to the classical causal hyperbolic propagation. The standard situation is that the two algebras in the definition of Einstein causality are equal; this situation is referred to as "Haag duality" [22].

But there exist models in which both causality requirements are fulfilled although Haag duality for algebras localized in multiply connected spacetime regions is violated; the simplest such regions are tori. The above calculation shows that this occurs for the operator algebras $\mathcal{A}$ generated by the free field strength $F_{\mu \nu}(x)$ of QED. The tori and the regularized surfaces were constructed at a fixed time, but using the causal completeness property, the relation (13) continues to hold for their causal completion $\mathcal{T}^{\prime \prime}, \hat{\mathcal{T}}$ " in spacetime when the separation in space passes to a space-like separation in spacetime.

The above observation about the existence of observables, which can be localized on arbitrary (regularized) surfaces which share the same boundary tori, can then be expressed as

$\vec{H}\left(\vec{\Phi}_{\hat{D}, \rho}\right) \subset \mathcal{A}^{\prime}\left(\hat{\mathcal{T}}^{\prime}\right)$ but not in $\mathcal{A}(\hat{\mathcal{T}})$.

The magnetic flux commutes with all operators in $\mathcal{A}(\mathcal{O})$, with $\mathcal{O}$ a bounded contractible spacetime region which does not intersect $\hat{\mathcal{T}}$. One can always change the surface, while keeping its boundary in such a way that it is outside $\mathcal{O}$, but the previous calculation shows that this is not possible if $\mathcal{O}$ is a interpenetrating torus.

An Aharonov-Bohm-like situation arises if one replaces $\hat{\mathcal{T}}$ by a two-sided infinitely extended tube (which can be viewed as a torus which closes at conformal infinity).

\footnotetext{
5 Isomorphisms between localized algebras in different spacetime dimensions violate the causal completeness properties on one side of the isomorphsim. This is the reason why the Maldacena conjecture (the claim that the mathematical AdS-CFT isomorphism relates two causally localizable QFTs) is incompatible with causality [12,26,27].
}

The use of string-local vector potentials in Hilbert space permits an elegant explicit construction of such dualityviolating operators. For this purpose one starts from the relation (3). In the massive case the loop integral over the string-local vector potential is equal to that of its point-like counterpart. In the zero mass limit the Proca potential and $\phi$ do not exist; but since the differences $\phi(x, e)-\phi\left(x, e^{\prime}\right)$ remains infrared finite ${ }^{6}$, the difference between two identical Wilson loop but with different string directions vanishes,

$\oint A_{\mu}(x, e) \mathrm{d} x^{\mu}-\oint A_{\mu}\left(x, e^{\prime}\right) \mathrm{d} x^{\mu}=0$.

This leads to a kind of topological $e$-dependence; the loop integral still "remembers" that there was a directional dependence, but "it forgets" the concrete space-like direction into which the $e$ pointed. This "topological memory" corresponds to the breakdown of Haag duality in QED.

One may picture this situation in terms of a semi-infinite cylinder formed by parallel space-like lines in the $e$ direction which emanate from the points on the Wilson loop and extend to infinity. By $\rho$-regularization one can convert the circle into a torus, in which case the wall of the cylinder has a finite thickness and the Wilson loop integral represents a well-defined operator to be used in localization arguments. The semi-infinite cylinder is the covariant substitute of the surface and the deformation of the latter corresponds to the Lorentz transformation of the former. A magnetic loop which passes through the electric Wilson loop has to penetrate the cylinder wall somewhere. As before, an infinite extended magnetic flux corresponds to a loop which closes at infinity.

This constructions of a regularized Wilson loop, using a massless string-local vector potential in Hilbert space, leads to an elegant concrete realization of an operator which is in $\mathcal{A}\left(\mathcal{T}^{\prime}\right)^{\prime}$ but not in $\mathcal{A}(\mathcal{T})$. Whereas the perturbation theory of the gauge invariant $S$-matrix and the local observables are correctly described, the gauge-invariant Wilson loop defined in terms of the point-like vector potential of gauge theory fails to account for the breakdown of Haag duality and the closely related Aharonov-Bohm effect (see below); the topological property is lost in gauge theory. This was well known to the authors of [25]. In the present formalism the vector potentials "live" in the same Hilbert space as the field strengths.

There exists another interesting scalar potential $\phi\left(x, e, e^{\prime}\right)$ $\equiv \int_{0}^{\infty} e^{\prime \mu} A_{\mu}\left(x+\lambda e, e^{\prime}\right) \mathrm{d} \lambda^{\prime}$, which depends on two string directions and vanishes on the diagonal $e=e^{\prime}$; hence it is localized on the space-like wedge region spanned by the two space-like half-lines $\mathbb{R}_{+} e, \mathbb{R}_{+} e^{\prime}$. The important observation is that the zero mass limit of suitable normalized exponential correlation functions of $\phi$ remain finite for $m \rightarrow 0$.

\footnotetext{
6 I am indebted to Jens Mund for calling attention to this difference between the massive case and its massless limit.
} 
This is reminiscent of the two-dimensional situation which one encounters with conserved currents. The curl of the related pseudo-current $\tilde{j}_{\mu}=\varepsilon_{\mu \nu} j^{v}$ vanishes so that it permits a representation in terms of a derivative of a scalar field $\tilde{j}^{\mu}=\partial^{\mu} \chi(x)$. Again its zero mass limit diverges and the exponential functions create new sectors in the massless limit.

The analogy may be described as follows:

$$
\begin{aligned}
\chi(x, e)= & \int_{0}^{\infty} \tilde{j}_{\mu}(x+\lambda e) e^{\mu} d \lambda \sim \phi\left(x, e, e^{\prime}\right) \\
= & \int_{0}^{\infty} A_{\mu}\left(x+\lambda e, e^{\prime}\right) e^{\mu} \mathrm{d} \lambda \\
& \exp i g \chi(x, e) \sim \exp i g \phi\left(x, e, e^{\prime}\right) .
\end{aligned}
$$

In both cases the zero mass limit of the exponentials generates new sectors of the operator algebras generated by $j_{\mu}$, respectively, $A_{\mu}(x, e)$. The exponentials of $\chi$ play an important role in creating "anyonic" sectors and "fermionization" in $d=1+1$. The exponential functions of $\phi$ on the other hand are expected to be important in a future theory of "infraparticles". 7 Another related shortcoming of the gauge theory in Krein space had been noticed in [28] namely that it leads to a vanishing Maxwell charge; something which one does not expect in the Hilbert space setting. We hope to return to this interesting problem.

Calculation in quantum mechanics are generally done in the Coulomb (or radiation) gauge. It is interesting to note that this non-covariant and non-local but rotation-invariant potential in Hilbert space results from the string-local potential by averaging over string directions $e$ in a space-like hypersurface. It plays no role in covariant renormalization theory, but most calculations in quantum mechanics (including those of the A-B effect) use it. The string-local vector potential is its local covariant counterpart.

The quantum string-local vector potential has a classical analog. It can be obtained in terms of the expectation value of the quantum string-local potential in coherent states. In this way the above operator calculations pass to their classical counterpart; as argued in the sequel this shows that the breakdown of Haag duality for free fields is basically a classical phenomenon in which the localized subalgebras are replaced by modular localized subspaces of the $(s=1, m=0)$ Wigner-particle space [11].

In the classical setting the algebra $\mathcal{A}(\mathcal{T})$ corresponds to $\mathcal{T}$ localized modular subspace $K(\mathcal{T})$ of the Wigner space; the dash on the localization region retains its geometric meaning, and the commutant of the algebra passes to the symplectic complement of the subspace $K(\mathcal{T})$ (which is defined in terms of the imaginary part of the inner product in Hilbert space

7 The infraparticle Dirac field is similar to the incoming free field, except that it has the softening of the electron mass-shell already built in.
[11]). The classical analog of the breakdown of Haag duality is a topological phenomenon in classical field theory. In this setting the $\mathcal{T}$ of the Aharonov-Bohm effect is described in terms of a classical magnetic flux through an infinitely extended solenoid which closes at conformal infinity. The role of quantum mechanics in the $\mathrm{A}-\mathrm{B}$ effect is the creation of the electric Wilson loop by splitting an electron beam. Note that the topological semi-infinite cylinder attached to the Wilson loop maintains a causal connection with the magnetic flux whereas the point-local vector potential has none.

The breakdown of the Haag duality and its classical analog disappears in case of massive vector mesons. Whereas the infrared properties are characteristic for interacting massless vector potentials, the duality violation is a pure kinematic effect. Such a topological duality violation occurs for all massless $s \geq 1$ tensor potentials.

The conformal invariance of the field strength raises the question whether instead of space-like strings it would not be more appropriate to work with light-like covariant stringlocal vector potentials; in this case the de Sitter differential geometry would be replaced by that of the boundary of the light-cone. Actually this could also lead to conceptual and computational simplifications. Basic relations, as those between point-local potentials and their string-local counterparts (the string-local potential and its scalar escort $\phi$ ) seem to be preserved. We hope to return to this interesting problem in a future publication.

Finally, it is worthwhile to mention that the use of stringlocal potentials also removes that "quirky feeling" about a missing causal relation between the Wilson loop formed and the magnetic field passing through it. This feeling disappears once one realizes that it is caused by the use of point-like vector potentials of gauge theory. This may be bad news for the popular literature, but it is certainly helpful for demonstrating the power of Hilbert space positivity (which, as shown before, even leaves its imprint in the form of topological properties on the classical limit).

\section{Perturbation theory in terms of string-local vector potentials}

More important for particle physics is the improvement of renormalizability through the use of the string-local formalism. The simplest illustration of this idea is provided by for massive QED. One rewrites the point-local nonrenormalizable interaction density into its string-local counterpart as follows:

$$
L^{P}=A_{\mu}^{P} j^{\mu}=\left(A_{\mu}-\partial_{\mu} \phi\right) j^{\mu}=L-\partial^{\mu}\left(j_{\mu} \phi\right)=L-\partial^{\mu} V_{\mu},
$$

$\int L^{P}=\int L \longleftrightarrow L^{P} \stackrel{A E}{\simeq} L$, adiabatic equiv. 
Here we have used the conservation of the free current (all fields are non-interacting) and the notation $L$ for the string-local interaction density. The power-counting violating point-like interactions have been written in terms of a $L$ with $d_{\text {sd }}^{\text {int }} \leq 1$ and a $d_{\text {sd }}=5$ derivative $\partial V$ term which, at least in models with a mass gap, can be disposed of in the adiabatic limit of the first order $S$-matrix (second line). The nontrivial step of generalizing this idea to higher order time-ordered products will be undertaken in the next section.

Not all models are that simple. An interaction of a massive vector meson with a Hermitian field $L^{P}=A^{P} \cdot A^{P} H$ leads to a $L, V$ pair $L-\partial^{\mu} V_{\mu}=L^{P}$ (omitting the shared coupling strength $g$ )

$$
\begin{aligned}
& L=m\left\{A \cdot(A H+\phi \overleftrightarrow{\partial} H)-\frac{m_{H}^{2}}{2} \phi^{2} H\right\} \\
& V_{\mu}=m\left\{A_{\mu} \phi H+\frac{1}{2} \phi^{2} \overleftrightarrow{\partial}_{\mu} H\right\} \\
& Q_{\mu}=d_{e} V_{\mu}=m u\left(A_{\mu} H+\phi \overleftrightarrow{\partial}_{\mu} H\right) .
\end{aligned}
$$

In this case the string-local interaction density (and not only $V_{\mu}$ as in massive QED) depends on $\phi$. There are other terms within the power-counting restriction which we could have added namely $c H^{3}+d H^{4}$ with initially independent coupling strengths $c, d$. But it turns out that the second and third order $e$-independence of the $S$-matrix induces these couplings anyhow, as well as additional $H-\phi$ couplings. The basic difference of this kind of induction as compared to the standard counterterm formalism is that it does not increase the number of parameters. In fact the first and second order induced $H$-self-couplings and $H-\phi$ couplings taken together will turn out to have the form of a Mexican hat potential. Its appearance has nothing to do with SSB; it is fully explained in terms of the $e$-independence of the $S$-matrix (next section).

Here the terminology induced refers to contributions whose presence is required for the $e$-independence of scattering amplitudes. This is a consequence of scattering theory in the presence of a mass gap; we refer again to a general theorem in [21,22], which states that the LSZ scattering theory permits an extension to string-local fields and that the difference between point- and string-localized fields disappears on the level of incoming/outgoing particle states. In the context of the gauge theoretical formalism in Krein space there are no physically localized fields in Hilbert space to which this theorem can be applied; hence the imposition of gauge invariance on the perturbative representation of the scattering amplitude remains a perturbative prescription.

The Krein space of gauge theory does not contain multiparticle Wigner states; the best one can do is to view the free fields $A^{P, K}$ as the substitute for the generating fields $A^{P}$ of the Wigner particles in the sense of (10). ${ }^{8}$ The field-particle connection resulting from scattering theory and problems concerning the relation of the elementary (model-defining) fields with the (generally composite) local observables and their associated bound states remain outside its physical range.

A useful reformulation for the construction of $e$ independent first order interaction densities in terms of a given number of string-local fields is to look directly for $L, V_{\mu}$ or $L, Q_{\mu}$ pairs with

$d_{e}\left(L-\partial^{\mu} V_{\mu}\right)=0 \quad$ or $\quad d_{e} L-\partial^{\mu} Q_{\mu}=0, \quad d_{\mathrm{sd}}^{L} \leq 4$,

instead of starting from an $L^{P}$. The $L$ of such a pair is then determined up to exact forms $d_{e} C$ and the $V_{\mu}$ up to a divergence-free current. The definition of point-like interaction densities in terms of such pairs is particularly useful for the generalization to higher order time-ordered point-local interaction densities.

Whereas for theories with a mass gap the construction of a $L, V_{\mu}$ pair and the extension to $e$-independent time-ordered higher order densities has a clear motivation, this is lost in the massless limit when $\phi$ and $V_{\mu}$ and the $S$-matrix cease to exist (become infrared divergent). This, as well as the appearance of unexpected new phenomena, as those presented in the previous section, leaves only one way to construct massless $s=1$ interacting fields. It consists in taking the $m \rightarrow 0$ limit of the correlation functions of the associated massive theory and construct the massless operator theory in Hilbert space from its correlation functions.

In this way one avoids the intermediate use of infrareddivergent operators as $V_{\mu}, Q_{\mu}$ and one also bypasses a confrontation with the (futile) problem of describing the zero mass Hilbert space in terms of limits of Wigner-Fock spaces. Wightman's reconstruction theorem ensures that a Hilbert space and quantum fields can be constructed from (positivityobeying) correlation functions [30]. To implement these ideas one must first extend the present formalism so that in addition to the $S$-matrix it also includes the construction of interacting fields.

The use of string-local charge-carrying matter fields is supported by rigorous results in QED [9]. Whereas in the presence of a mass gap point-local matter fields may still exist as singular fields with unbounded short-distance dimensions (and therefore outside the setting of localizable Wightman fields), these non-perturbative results excludes such possibilities in the massless limit.

The BRST gauge formalism uses charge-carrying unphysical point-like fields. This limits its physical range to the

\footnotetext{
8 The speculative remarks in [29] on the gauge-theoretic substitutes of Wigner particles acquire a concrete meaning in the present setting.
} 
construction of the perturbative $S$-matrix. ${ }^{9}$ Its perturbative construction has been presented in [4]. It can be formulated in a setting which parallels the $L, Q$ pairs,

$\mathfrak{s} L^{K}-\partial^{\mu} Q_{\mu}^{K}=0$,

$S=\int L^{K}(x) \mathrm{d}^{4} x, \quad \mathfrak{s} S=0$

where $K$ refers to Krein space. Integration over Minkowski spacetime removes the $Q$ contribution in the adiabatic limit so that the $S$-matrix only depends on $L^{K}$. The formal expressions in the case of coupling to a Hermitian matter field $H$ parallel those of (19) with the fields replaced by their Krein space counterparts.

The BRST formalism, apart from the vacuum sector generated by gauge invariant observables, implements only onshell unitarity. Gauge theory can describe scattering, but it does not permit to extend on-shell unitarity to off-shell positivity; properties abstracted from gauge-dependent fields have no physical content. In most applications of QFT to particle physics the $S$-matrix covers all problems of interests. But in massless $s=1$ theories as QED, for which the $S$-matrix ceases to exist, one is forced to use momentumspace recipes for the scattering of charge-carrying particles in terms of photon-inclusive cross sections.

A full spacetime understanding of "infraparticles" in QED does not yet exist, not to mention the much harder problem of confinement of gluons and quarks in QCD. Such problems are outside the range of gauge theory. They require the understanding of large-distance properties of fields. For this one needs to extend the unitarity of the $S$-matrix ("on-shell unitarity") to the Hilbert space description ("off-shell unitarity") of fields. This cannot be achieved within the setting of point-local fields since for interactions involving vector mesons such a description is incompatible with the Hilbert space positivity.

As described before, the way out is to use the structural simplicity of a Wigner-Fock Hilbert space description in the presence of a mass gap and to define the massless theory in terms of the $m \rightarrow 0$ limit of the correlation functions. In this way one maintains the positivity in the massless limit so that one can study the long-distance behavior in terms of physical string-local fields.

A particularly interesting case arises if confinement in QCD is realized in terms of the vanishing of those correlation functions which contain besides point-like composites and "string-bridged" $q \bar{q}$ pairs also string-local gluons and quarks. In that case one obtains a QFT in which the basic fields, used in the definition of the perturbative first order interaction density, have disappeared and only their point-

\footnotetext{
${ }_{9}$ In the present paper this terminology refers to the physical on-shell scattering operator and not to Bogoliubov's generating off-shell $S$ functional (from which the $S$-matrix arises in the adiabatic limit).
}

local composites (gluonium, hadrons) remain. The only way to describe such a situation within our present understanding of QFT is to view it as a massless limit (see the last section).

An analog of (3) for arbitrary spin exists for all $s \geq 1$; instead of string-local scalar $\phi$ one obtains a linear relation involving derivatives of string-local escort fields $\phi$ of spin $<s$ with short-distance dimension $d_{\mathrm{sd}}<s+1$. It is not clear whether this can be used to generalize the idea of obtaining short-distance improving $L, \partial V$ pairs to higher spin. A particularly interesting case is $s=2$.

\section{Induced higher order contributions and the Higgs model}

The construction of a string-local renormalizable first order interaction density in terms of a $L, V_{\mu}$ pair permits an extension to higher orders. The second order relation $\left(L^{\prime}\right.$ stands for $L\left(x^{\prime}, e^{\prime}\right)$ )

$\left(d_{e}+d_{e^{\prime}}\right)\left(T L L^{\prime}-\partial^{\mu} T V_{\mu} L^{\prime}-\partial^{\prime \nu} T L V_{v}^{\prime}+\partial^{\mu} \partial^{\prime \nu} T V_{\mu} V_{\nu}^{\prime}\right)=0$

would automatically follow from the first order relation (17) if it would not be for the singularities coming from timeordering of distributions. ${ }^{10}$ If this renormalization condition can be implemented, the first order definition (20) of the point-like interaction density permits a second order generalization,

$T L^{P} L^{P \prime}:=\left(T L L^{\prime}-\partial^{\mu} T V_{\mu} L^{\prime}-\partial^{\prime \nu} T L V_{\nu}^{\prime}+\partial^{\mu} \partial^{\prime \nu} T V_{\mu} V_{\nu}^{\prime}\right)$,

and one could hope to be able to generalize this idea to higher orders.

It is instructive to recall how the CGI gauge-theoretic procedure [4] deals with this problem. In that case one uses the weaker " $Q$-version"

$\mathfrak{s} T L^{K} L^{K^{\prime}}-\partial^{\mu} T Q_{\mu}^{K} L^{K^{\prime}}-\partial^{\prime \mu} T L^{K} Q_{\mu}^{K^{\prime}}=0$.

Replacing the BRS $\mathfrak{s}$ by $d=d_{e}+d_{e^{\prime}}$ and omitting the superscript $K$, one obtains the corresponding SLF relation. Although this $Q$-relation is weaker than the $V$-relation, it suffices to define a second order gauge-invariant $(\mathfrak{s} S=0)$ or string-independent $(d S=0) S$-matrix, since the derivative terms drop out in the adiabatic limit.

Starting from a time-ordering $T_{0}$ in which all derivatives of fields are taken outside, one can use the Epstein-Glaser

\footnotetext{
$\overline{10}$ A systematic presentation of the differential form calculus will be contained in forthcoming work by Mund.
} 
minimal scaling restriction to define renormalized parameterdependent $T$-products. For a scalar free field of scale dimension $d_{\mathrm{sd}}=1$ the $T$-ordering is

$$
\begin{aligned}
& \left\langle T_{0} \partial_{\mu} \varphi(x) \partial_{\nu}^{\prime} \varphi^{*}\left(x^{\prime}\right)\right\rangle=\partial_{\mu} \partial_{\nu}^{\prime}\left\langle T_{0} \varphi(x) \varphi^{*}\left(x^{\prime}\right)\right\rangle \\
& \left\langle T \partial_{\mu} \varphi(x) \partial_{\nu}^{\prime} \varphi^{*}\left(x^{\prime}\right)\right\rangle=\left\langle T_{0} \partial_{\mu} \varphi(x) \partial_{\nu}^{\prime} \varphi^{*}\left(x^{\prime}\right)\right\rangle+c g_{\mu \nu} \delta\left(x-x^{\prime}\right),
\end{aligned}
$$

and the second line is the definition of a one-parametric $T$ ordering according to the $\mathrm{E}-\mathrm{G}$ minimal scaling rule. It turns out that this freedom can be used to absorb certain "anomaly" contributions into a redefinition of time-ordering. The strategy is to use the freedom in the definition of $T$ in such a way that (25) is fulfilled.

One defines an anomaly as a measure of the violation of (23) or (25) if one uses $T_{0}$ instead of the still unknown $T$. We have

$$
\begin{aligned}
\mathfrak{A}_{V}:= & \left(d_{e}+d_{e^{\prime}}\right)\left(T_{0} L L^{\prime}-\partial^{\mu} T_{0} V_{\mu} L^{\prime}-\partial^{\prime \nu} T_{0} L V_{v}^{\prime}\right. \\
& \left.+\partial^{\mu} \partial^{\prime \nu} T_{0} V_{\mu} V_{v}^{\prime}\right), \\
\mathfrak{A}_{Q}:= & \left(d_{e}+d_{e^{\prime}}\right) T_{0} L L^{\prime}-\partial^{\mu} T_{0} Q_{\mu} L^{\prime}-\partial^{\prime \mu} T_{0} L Q_{\mu}^{\prime}=0 .
\end{aligned}
$$

For the calculation of two-particle scattering we only need the 1-particle contraction component (the "tree" approximation).

The simplest nontrivial illustration is provided by massive scalar QED. ${ }^{11}$ In that case the presence of a derivative in the current (17) leads to delta function contributions from the divergence of the two-point function of the charged field $\varphi$,

$$
\partial^{\mu}\left\langle T_{0} \partial_{\mu} \varphi(x)\left(\partial_{\nu}^{\prime}\right) \varphi^{*}\left(x^{\prime}\right)\right\rangle=\left(\partial_{\nu}\right) \delta\left(x-x^{\prime}\right)+\text { reg. }
$$

where the regular part comes from the use of the free field equation inside the $T_{0}$. This. together with $d_{e}(\partial \phi \cdot A)=$ $\frac{1}{2} d_{e}(A \cdot A)$, which results from the application of $d_{e}$ to (3), yields [19]

$\mathfrak{A}_{Q}=d_{e} T_{0} L L^{\prime}-d_{e} N_{\mathrm{sym}}+\partial_{\mu} N_{\mathrm{sym}}^{\mu}$,

$N_{\text {sym }}=N+N^{\prime}, N_{\text {sym }}^{\mu}=N^{\mu}+N^{\mu \prime}$,

$N=\delta\left(x-x^{\prime}\right) \varphi^{*} \varphi A \cdot A^{\prime}, N_{\mu}=\delta\left(x-x^{\prime}\right) \varphi^{*} \varphi \phi A_{\mu}^{\prime}$.

The $N^{\prime}$ and $N_{\mu}^{\prime}$ are obtained by symmetrization $x, e \longleftrightarrow$ $x^{\prime}, e^{\prime}$. Using the freedom (26), $N$ and $N_{\mu}$ can be absorbed into a redefinition of the time-ordering,

$$
\begin{aligned}
T L L^{\prime} & =T_{0} L L^{\prime}+N_{\mathrm{sym}}, T Q_{\mu} L^{\prime}+T L Q_{\mu}^{\prime} \\
& =T_{0} Q_{\mu} L^{\prime}+T_{0} L Q_{\mu}^{\prime}+N_{\mu, \mathrm{sym}}, \\
S^{(2)} & =-\frac{g^{2}}{2} \iint T L L^{\prime} \mathrm{d}^{4} x \mathrm{~d}^{4} x^{\prime} .
\end{aligned}
$$

In this way the SLF counterpart $d S=0$ of (25) has been established. The derivation of the second order point-like density (24) is more involved but it does not change $T L L^{\prime}$.

11 The renormalization theory of massive spinor QED has no anomalies.
The result is hardly surprising, since the presence of a quadratic term in the vector potential from $N_{\text {sym }}$ to the second order is well known from gauge theory. But there is a subtle point of fundamental significance which should be mentioned. In general it is not possible to set $e=e^{\prime}$. The reason is that string-local fields $\Psi(x, e)$ fluctuate in both $x$ and $e$, and hence products of fields with the same $e$ do not make sense. Fortunately, Wick-ordered products do not only permit the $x$ to coincide, but they also allow $e^{\prime} s$ to coalesce. Such $e^{\prime} s$ will be called "mute". But the expansion of time-ordered products into Wick-products also involves time-ordered contractions for which the $e$-fluctuations prevent $e^{\prime} s$ from coinciding.

Consider the propagator of a string-local vector meson:

$$
\frac{1}{p^{2}-m^{2}}\left(-g_{\mu \nu}+\frac{p_{\mu} p_{\nu}}{(p \cdot e-i \varepsilon)\left(p \cdot e^{\prime}+i \varepsilon\right)}+\cdots\right) .
$$

As a result of the different $i \varepsilon$-prescriptions, the distributional boundary values are ill-defined for $e=e^{\prime}$. This is a problem which cannot be solved by renormalization theory. It explains why the axial gauge (which treats the $e$ as a rigid gauge parameter) had to fail.

In the present context the $e^{\prime} s$ are fluctuation spacetime variables of a string-local covariant field. The implementation of the independence of the $S$-matrix of the string directions $d S=0$ based on the differential calculus of the $1+2$ dimensional de Sitter space guarantees that this problem disappears after adding sufficiently many on-shell contributions. The simplest illustration is that of second order scattering of charged particles. In that case the dangerous contribution comes from the vector meson propagator (34). But the contribution containing both $e^{\prime} s$ disappears after the use of the onshell current conservation ${ }^{12}$ and the remaining $e$-dependence is then canceled by the contribution from $N_{\mathrm{sym}}$.

It is not necessary to go into the details of these cancelations, since the relation $d S=0$, which follows in the adiabatic limit from (32), guaranties $e$-independence. But as individual perturbative contributions to gauge-invariant scattering amplitudes in gauge theories are generally not gauge-invariant, it is hardly surprising that individual contributions in the SLF setting still depend on $e$. What is, however, somewhat unexpected is that such contributions may diverge for coalescent $e^{\prime} s$. The differential calculus in the $1+2$-dimensional de Sitter space of string directions only guaranties that the sum of perturbative contributions in a particular order converges. A formal off-shell continuation by hand would destroy this result; only the extension of perturbation theory to fields can preserve the independence from $e$-fluctuations resulting from inner propagators.

\footnotetext{
12 The argument parallels that of second order gauge invariance in gauge theories.
} 
This observation contains an important message. The positivity of Hilbert space, which required the use of string-local fields in order to preserve renormalizability, can be implemented in such a way that the scattering matrix remains string-independent. But in contrast to gauge theory individual contributions to $S$ may depend on the $e^{\prime} s$ in such a way that equating different $e^{\prime} s$ causes infinite fluctuations.

On the basis of these observations one anticipates that an extension of this formalism to interacting string-local fields will lead to perturbative contributions which depend on the individual string directions of inner propagators after all $p$ integration have been carried out. But one expects that in suitable sums over the contributions the dependence on the inner $e^{\prime} s$ disappears so that only the possible string dependence of the interacting fields remains. This is very different from gauge theory; the BRST $\mathfrak{s}$ is a global operation which cannot distinguish between internal and external propagators.

The Hilbert space setting, which requires the use of the differential calculus in the directional de Sitter space, is certainly more elaborate than the gauge formalism, but unlike the latter it is not limited to the construction of local observables and the $S$-matrix but it promises the construction of all fields.

After this interlude of conceptual difference of the SLF setting with operator gauge theories presented in the contest of second order scalar massive QED, we now turn to the problem of the second order calculation of the $A-H$ coupling (19). Up to $H$ self-interactions the $L, V$ pair is uniquely determined by first order power-counting $d_{\text {sd }}^{\text {int }} \leq 4$ and the requirement $d_{e}(L-\partial V)=0$.

Our aim is to highlight differences between the gaugetheoretic second order calculations as it was presented in $[4,5]$ and the SLF setting. In that work the calculation of the second order gauge-invariant for the Higgs model $S$-matrix starts with a first order $L^{K}, Q_{\mu}^{K}$ pair which is identical to (19), except that $L, A_{\mu}, \phi$ and $Q_{\mu}$ now have the superscript $K$ and instead of the geometric differential calculus one now has the abstract $\mathfrak{s}$-operation. The renormalizable $c H^{3}+d H^{4}$ terms, which were already mentioned after (19), turn out to be necessary in order to keep the third order tree contributions free of anomalies; beyond the third order there are no tree anomalies.

The result is [4]

$$
\begin{aligned}
& T_{0} L^{K} L^{K \prime}+i \delta\left(x-x^{\prime}\right)\left(A^{K} \cdot A^{K} H^{2}+A^{K} \cdot A^{K} \phi^{2}\right) \\
& \quad-i \delta\left(x-x^{\prime}\right) R^{K} \\
& R^{K}=-\frac{m_{H}^{2}}{4 m^{2}}\left(\phi^{K, 2}+H^{2}\right)^{2}, \\
& V^{K}=g^{2} \frac{m_{H}^{2}}{8 m^{2}}\left(H^{2}+\phi^{K, 2}+\frac{2 m}{g} \phi^{K}\right)^{2}-\frac{m_{H}^{2}}{2} H^{2}
\end{aligned}
$$

where $V^{K}$ is the contribution to the second order $S$-matrix which results from combining the second order $R^{K}$ with the first order $H$-self-interactions. The appearance of a $1 / g$ term shows that this way of writing is somewhat artificial, but it permits us to relate the physical parameters $m, m_{H}, g$ to those which result from applying a shift in field space (the abelian SSB Higgs mechanism) to the gauge-dependent scalar field of QED.

All interactions which involve point-like vector mesons require the setting of gauge theory, including those in which the vector meson interacts with Hermitian fields. The result (35) shows that there is no place for SSB. Whereas this is possible for self-interacting scalar fields, it is ruled out when scalar fields also couple to vector mesons. In that case the vector mesons require the implementation of the rules of gauge theory, which leaves no room for SSB. The coupling to stringlocal vector potentials in the SLF Hilbert space formulation converts the point-local scalar fields into interacting stringlocal fields whereas SSB is limited to point-local scalar fields.

This is confirmed by the second order ghost-free SLF Hilbert space setting which only uses the causal localization principles of QFT. Instead the abstract cohomological BRST formalism it is based on the geometric differential form calculus in the de Sitter space applied to string-local fields with fluctuating space-like directions. The calculation of a $e$-independent $S$-matrix follows similar steps; the details will be presented elsewhere.

There is, however, one difference between the gaugetheoretic calculation and its SLF analog which is worthwhile mentioning. In addition to the induced potential $R$, there is a second order term of the form

$\delta\left(x-x^{\prime}\right) A_{\mu}^{P} A^{\mu \prime} u\left(\phi^{\prime}-\phi\right)+\left(e \longleftrightarrow e^{\prime}\right)$.

Since the short-distance dimension of the point-local Proca field is $d_{\mathrm{sd}}^{P}=2$, this contribution has $d_{\mathrm{sd}}=5$ and hence violates the power counting bound. But since it vanishes for $e=e^{\prime}$ it causes no problem for the $e$-independent $S$-matrix.

The Schwinger-Swieca screening effect, which asserts that any interaction which involves massive vector mesons (independent of whether it couples to charged or Hermitian matter) leads to a vanishing Maxwell charge, is a rigorous argument against any form of SSB. It generalizes the screening of the Maxwell current of a free massive field strength,

$j_{v}:=\partial^{\mu} F_{\mu v}=m^{2} A_{v}^{P}, \quad Q_{\operatorname{Max}}=\int A_{0}^{P}(x) \mathrm{d}^{3} x=0$,

which can easily be checked by looking at the form of the two-point function of the free Proca field. Higher order contributions to $F_{\mu \nu}$ require an extension of the $S$-matrix formalism to interacting fields, but thanks to the Schwinger-Swieca theorem, this is not needed if one only wants to exclude a SSB mechanism.

If the Higgs model would be the result from a SSB, the charge would diverge $Q=\infty$ instead of vanishing. 
This is part of the definition of SSB. A symmetry is called "spontaneously broken" if the Noether theorem cannot be inverted, i.e. when a conserved current $\partial^{\mu} j_{\mu}=0$ leads to a divergent charge (the generator of a symmetry), formally $Q=\int j_{0}(x) \mathrm{d}^{3} x=\infty$.

A SSB model is closely related to a symmetric theory, in fact both are unitarily inequivalent representations ("different vacua") of the same local observables algebras [31]. The shift in field space is a quasiclassic trick which prepares the first order interaction of a SSB theory starting from a symmetric interaction. Only field shifts which lead to largedistance diverging charges implement SSB. If the charge is screened, as in the Higgs model, the field shift manipulation has no relation to the intrinsic properties of the model and if the resulting model turns out to be consistent one is required to look for a different explanation of its physical properties.

The most interesting situation is that of massive selfinteracting vector mesons. A first order $L^{K}, Q_{\mu}^{K}$ pair within the power-counting limit is easily found, but it is not possible to maintain this renormalizability restriction in the calculation of the second order gauge-invariant $S$-matrix. As already mentioned in the introduction, one can only compensate the "bad" terms by introducing a first order coupling with scalar $H$-fields which in second order produces a compensating bad terms. The calculations have been carried out in the CGI setting of gauge theory in [4,5]; these authors also showed that in the case of just one $H$ the model contains no additional parameters besides one coupling strength and the masses of $H$ and the vector mesons. In [6] this situation was reviewed and compared with the actual situation of the Standard Model.

This result is confirmed in the SLF Hilbert space setting. The details require more extensive calculations and will be presented in a joint paper with Jens Mund. There is one difference with the CGI calculation which is worth mentioning. The calculation of the second order $S$-matrix from the first order $A-H$ coupling

$$
L_{H}=\sum_{a, b} d_{a, b}\left(A_{\mu, a}^{P} A_{b}^{\mu} H+A_{a}^{\mu} \phi_{b} \partial_{\mu} H-\frac{1}{2} m_{H}^{2} \phi_{a} \phi_{b}\right)
$$

parallels that of the abelian coupling, but instead of the term (36), which has short-distance dimension $d_{\mathrm{sd}}=5$ instead of 4 (but fortunately vanishes on the $e$-diagonal $e^{\prime}=e$ ), one now finds

$$
\begin{aligned}
& \delta\left(x-x^{\prime}\right) \sum_{a, b ; a^{\prime} b^{\prime}} d_{a, b} d_{a,^{\prime} b^{\prime}} u_{a} A_{b^{\prime}}^{\mu}\left(A_{\mu, b}^{P} \phi_{a^{\prime}}^{\prime}-A_{\mu, a^{\prime}}^{P} \phi_{b}\right) \\
& \quad+\left(e \longleftrightarrow e^{\prime}\right),
\end{aligned}
$$

which does not vanish on the $e$-diagonal. Hence the second order contribution to the $e$-independent $S$-matrix contains a power-counting-violating term which can only be compensated by a similar term from the second order self-interaction between the massive vector mesons. The full calculation in the new Hilbert space setting will be contained in a forthcoming work.

As long as one considers the shift in field space and the subsequent gauge transformation as formal device which relates the well-known Lagrangian of scalar QED with a new Lagrangian in which a massive vector meson interacts with a H-field, no harm is being done. The problem only starts if one attributes a physical interpretation with this recipe. In this case one misses a new physical mechanism namely the loss of second order renormalizability of self-interacting massive vector mesons which can only be saved by extending the first order interaction by an additional coupling with a $H$-field which produces second order compensating terms.

Such a compensatory mechanism between fields with different spin which preserves renormalizability was hoped for to occur in models with supersymmetric couplings. Whereas such a requirements played no role in the definition of supersymmetry, it is the raison d'être for the $H$-particle. Formal manipulations of Lagrangians contain generally no physical information; the physical content of a model in QFT is always related to intrinsic properties of the model independent of the way in which it was constructed.

Terminologies as "fattening" or "being eaten" should have served as a warning that one is entering metaphoric swampland and reminded particle theorists that understanding of properties of QFT means connecting them with the foundational causal localization principle of QFT. We leave it to the historians to explain why, despite the correct terminology "Schwinger-Higgs screening" in some publications at the time of the Higgs paper the related ideas where lost in the maelstrom of time.

One of the reasons which contributed to its popularity may be related to the fact that the Higgs mechanism was discovered in at least three independent papers with identical metaphors about the Goldstone boson "being eaten by the massless vector meson". The problem here is not that important discoveries have been made by metaphoric ideas; this happened many times in the history of physics. What is, however, a bit embarrassing is that even 40 years later these reasonings appear in important documents.

For the sustentation of the impressive experimental effort, which after decades of search finally led to the discovery of $H$ at LHC, the narrative about particles, which in addition of generating masses of vector mesons also create their own mass, may have been a blessing; it would have been much harder to convince experimentalists that the fate of the Standard Model depends on the need to find an additional particle in order to uphold second order renormalization. The fundamental aspect of such an observation is more concealed since it is related to the not yet sufficient understood connection 
between renormalizability with the causal localization principle in a Hilbert space setting.

\section{Spacetime origin of the Lie structure of self-interacting vector mesons}

One of the unsolved problems of $s=1$ interaction is the understanding of the conceptual origin of the quantum Lie structure in models of self-interacting vector mesons. The answer cannot be given by referring to gauge theory. In classical gauge theory the Lie-algebra structure is part of the fiber bundle formalism whereas the only principle of QFT is causal localizability. The BRST gauge setting is a compromise between the ideas underlying classical gauge invariance and the quantum requirements. A derivation of the quantum Lie structure within the BRST gauge setting, as can be found in [4], is hardly surprising. It should rather be understood as a consequence of the foundational causal localization properties for self-interacting $s=1$ fields.

Quantum gauge symmetry cannot be realized in a Hilbert space; the preservation of the classical gauge structure requires the use of an indefinite metric Krein space and hence the gauge-theoretic derivation of the Lie-algebraic structure in a BRST gauge setting involves the use of a circular argument.

The understanding of the physical concepts behind local gauge symmetry turned out to be one of the hardest problems of local quantum physics. The origin of global gauge symmetries (inner symmetries) had already been well understood during the 1970s in terms of the DHR and the more general later DR superselection theory [22]. These constructions show that observable algebras, which typically arise as invariant subalgebras of field algebras under the action of a global symmetry group, contain sufficient information for reconstructing the symmetry group and the field algebra on which it acts.

This construction is somewhat astonishing since at first glance the causal localization properties of local observables seem to have no connection with group representation theory; the net of localized observable algebras is covariant under the Poincaré group (including the TCP operation) but it is no relation to gauge symmetry, be it global or local. Yet the classification of unitarily inequivalent local representations of the spacetime-indexed set of local algebras (the local superselection sectors of the observable algebra) leads indeed to a field algebra and a compact groups acting on it. The construction uses only the spacetime causal localization properties; the group theory is hidden in the composition structure ("fusion rules") of the localization-preserving inequivalent representations (endomorphisms) of the observable algebra [22]. In this way the origin of global gauge symmetries (inner symmetries) is fully accounted for.
These methods work in the presence of a mass gap but fail for interacting theories involving massless vector potentials. Local gauge symmetry tries to imitate global gauge symmetry at the price of losing the Hilbert space. There have been attempts to classify representation sectors of the algebra of local observables of QED [32]. They require the introduction of new concepts and their results are presently incomplete and far from being useful for the understanding of the problems of interacting massless vector mesons.

An understanding of the Lie group structure of selfinteraction vector mesons would be a useful step in this direction. Self-couplings between $s<1$ fields are not subject to such restrictions. Forming renormalizable trilinear and quadrilinear couplings between a finite set of low spin fields allows a very large number of independent coupling parameters. For any symmetric low spin $s<1$ model with one coupling parameter there exists a large number of less symmetric models with many independent couplings between the same free fields. So why is this not possible for $s=1$ ? The answer is that for $s \geq 1$ the Hilbert space positivity together with renormalizability is more restrictive.

Instead of an interaction density $L$ one has to find an $e$ dependent $L, V_{\mu}$ pair which satisfies a differential relation in $e$. The local counterterms of the inductive Epstein-Glaser formalism, which for $s<1$ interactions between pointlocal fields are only subject to the the appearance of pointlike counterterms at coalescent $x$ restricted by the minimal scaling requirement, have now to fulfill additional differential relations from string-crossings. These additional restrictions originate from maintaining causality in the presence of string-local field; they are not imposed symmetries. This more restrictive setting accounts for the appearance of Liealgebraic structures in interactions involving $s=1$.

The formalism of local gauge theory on the other hand results from trying to emulate this new structure within a point-local setting. The price is the loss of the Hilbert positivity and the "gain" is the promotion of the Lie-algebra structure of the coupling of fields to a full "local gauge symmetry". In this way the formalism is formally reunited with that for $s<1$ interactions at the price of its smaller physical range. Important problems of QED and QCD, which require the understanding of long-distance behavior of fields, remain outside the physical range of local gauge theory.

These new ideas are presently limited to perturbation theory. But they suggest that the non-perturbative construction of appropriately defined string-local field algebras, which are uniquely associated with the local observable algebras of massless $s=1$ interactions, may be a realistic goal of a project as that in [32]. This requires one to extend the pointlocal Wightman setting to string-local fields $\Psi$ (x.e) which are tempered distributions in both $x$ and $e$.

As in previous second order calculations one expects that the formal similarity with the gauge formalism in [4] extends 
to the model of self-interacting vector mesons. The following calculations show that this is indeed the case.

For simplicity we restrict our calculation to the massless first order interaction. The starting point is the renormalizable $L, Q_{\mu}$ pair

$L=\sum f_{a b c} F^{a . \mu v} A_{\mu}^{b} A_{v}^{c}, f$ antisymmetric

$Q_{\mu}=2 \sum f_{a b c} u_{\mu}^{a} F^{b, \mu v} A_{v}^{c}$.

As in all previous calculations operator products of free fields are always Wick-ordered. One expects that the Lie-algebra restriction for the $f$-couplings results from the imposition of second order $e$-independence.

$$
\begin{aligned}
\mathfrak{A}_{\mathrm{sym}}= & \mathfrak{A}+\mathfrak{A}\left((x, e) \longleftrightarrow\left(x^{\prime}, e^{\prime}\right)\right), \\
\mathfrak{A}= & d_{e} T_{0} L L^{\prime}-\partial^{\mu} T_{0} Q_{\mu} L^{\prime} \\
= & 2 \sum f_{a b c} u^{b} A_{\nu}^{c}\left\{f_{d e f} \delta_{\kappa, \lambda}^{\nu} \delta^{a d} A^{\prime d, \kappa} A^{\prime e, \lambda}\right. \\
& \left.+f_{d a f} \delta_{\lambda}^{v} F^{\prime d, \kappa \lambda} A_{\kappa}^{\prime f}+f_{\text {dea }} \delta_{\kappa}^{\nu} F^{\prime d, \kappa \lambda} A_{\lambda}^{e \prime}\right\} .
\end{aligned}
$$

Here the deltas are the singular parts (s.p.) which result from derivatives applied to $T_{0}$ propagators,

$$
\begin{aligned}
\delta_{\kappa, \lambda}^{\nu}(\xi) & =s \cdot p \cdot \partial_{\mu}\left\langle T_{o} F^{b, \mu \nu}(x) F_{d, \kappa \lambda}\left(x^{\prime}\right)\right\rangle \\
& \left.=-i\left(g^{\nu \lambda} \partial^{\prime \kappa}-g^{\nu \kappa} \partial^{\prime \lambda}\right) \delta(\xi)\right) \delta^{b d}, \\
\delta_{\lambda}^{\nu}(\xi) & =s \cdot p \cdot \partial_{\mu}\left\langle T_{0} F^{b, \mu \nu}(x) A_{a \lambda}\left(x^{\prime}\right)\right\rangle \\
& =-i g_{\lambda}^{\nu} \delta(\xi)-i e^{\prime \nu} \partial_{\lambda}^{\prime} \int_{0}^{\infty} \mathrm{d} s \delta\left(\xi-s e^{\prime}\right) .
\end{aligned}
$$

The second line contains a contribution from string crossing; the $s$-integral results from the Fourier transformation of the integrands (8),

$$
\frac{1}{p^{2}-m^{2}} \frac{e^{\prime v} p_{\lambda}}{p e^{\prime}-i \varepsilon}
$$

in the $\left\langle T F A^{\prime}\right\rangle$ propagators. In writing the first line we followed Scharf (page 113 in [4]) by using the freedom of a normalization term (according to the Epstein-Glaser scaling rules) in the various 2 -derivative contributions to the timeordered two-point functions in $\left\langle T F F^{\prime}\right\rangle$ e.g.

$\partial^{\mu} \partial^{v} D\left(x-x^{\prime}\right) \rightarrow \partial^{\mu} \partial^{v} D\left(x-x^{\prime}\right)+\alpha g^{\mu v} \delta\left(x-x^{\prime}\right)$.

At the end of the calculation the remaining anomaly must of course be independent of $\alpha$.

Symmetrizing in order to obtain $\mathfrak{A}_{s y m}$ one notices that for $e=e^{\prime}$ the string-local delta contributions cancel and one arrives at

$$
\begin{aligned}
& \mathfrak{A}_{\mathrm{sym}}=f_{a b c} u_{a} A_{c, v} \\
& \quad \times\left[f_{b e f} A_{e, \sigma} \partial^{\sigma} A_{f}^{v}+f_{d b f} A_{d, \rho} \partial^{v} A_{f}^{\rho}\right] 2 \delta\left(x-x^{\prime}\right)
\end{aligned}
$$

$$
\begin{aligned}
& +f_{a b c} f_{d e b}\left[(\alpha+1)\left(\partial^{\sigma} u_{a} A_{c, v}+u_{a} \partial^{\sigma} A_{c, v}\right) A_{d}^{v} A_{e, \sigma}\right. \\
& +(\alpha-1) u_{a} A_{c, v}\left(\partial^{\sigma} A_{d}^{v} A_{e, \sigma}+A_{d}^{v} A_{e, \sigma}\right] \delta\left(x-x^{\prime}\right) .
\end{aligned}
$$

The cancelation of the anomalies leads to $\alpha=1$ and the term $\partial^{\sigma} u_{a} A_{c, v} A_{d}^{v} A_{e, \sigma}$ has the form

$d_{e} N_{2}, N_{2}=\frac{1}{4} f_{a b c} f_{d e c} A_{a, \mu} A_{d}^{\mu} A_{b, v} A_{e}^{v} \delta\left(x-x^{\prime}\right)$.

The validity of the Jacobi identity is then a consequence of the remaining cancelation.

Another more systematic bookkeeping (following the logic of $\mathfrak{A}_{\text {sym }}$ in Sect. 3) would consist in converting derivatives of delta functions $\partial_{\mu} \delta \ldots$ into $\partial_{\mu}(\delta \ldots)-\delta \partial_{\mu}(\ldots){ }^{13}$ But here we followed Scharf in order to emphasize the formal proximity to the gauge formalism despite the conceptual differences between CGI and SLF.

Actually this derivation of the Lie-algebra structure of the second order interaction density involved a bit of cheating, since the operator $Q_{\mu}$, which was used in intermediate steps, has no zero mass limit. Such perturbative infrared divergencies are a warning against calculating directly in the massless theory, instead of constructing it from the $m \rightarrow 0$ limit of massive correlation functions.

The analogous calculations in the massive model avoids these problems. But as a consequence of the appearance of a compensatory renormalization-preserving $H$-field it is more involved. The verification of its Lie structure will be left to a joint publication with Jens Mund.

\section{Resumé and outlook}

The SLF setting extends the Hilbert space description of point-local $s<1$ interaction to $s \geq 1$ string-local interactions in which only the local observables remain pointlocal. Since it is a Hilbert space formalism, the powerful non-perturbative tools of functional analysis and operator algebras remain available. Gauge theory keeps the simpler point-local formalism for $s=1$ at the price of losing off-shell unitarity. Since the functional and operator algebra tools are not available in Krein space there is no non-perturbative control. The physical content is restricted to combinatorial perturbative constructions; conceptual and mathematical aspects concerning the relation between particles and fields remain outside its range.

The string-local fields $\Psi(x, e)$ are Wightman fields in both variables; they have to be smeared with Schwartz test functions both in Minkowski spacetime and in the de Sitter space of space-like directions $e$. The differential geometry and the associated differential form calculus of $d=1+2$ dimensional de Sitter space is a spacetime analog of the

\footnotetext{
13 This was pointed out to me by Jens Mund.
} 
abstract BRST cohomology of gauge theory defined in terms of the nilpotent $\mathfrak{s}$ operation (the ghost formalism).

The continued validity of the appropriately adjusted LSZ or Haag-Ruelle scattering theory $[21,22]$ in the presence of a mass gap permits to identify the Hilbert space with a WignerFock space in which scattering theory relates the Wigner particles to the interacting fields. For interactions involving massless vector mesons this field-particle relation breaks down and the $S$-matrix is lost; the fields are no longer connected with Wigner particles. In the perturbative SLF Hilbert space setting this manifests itself right from the beginning. A first order interaction $L$ cannot be defined since a scalar string-local $\phi$ escort field, and hence also $V_{\mu}$ and $Q_{\mu}$, have no massless limit. In the following we will argue that this loss is actually an asset, since it points into new directions for solving problems which are outside the scope of gauge theory.

The loss of the interaction density and a unitary $S$-matrix in the massless limit is not the result of a shortcoming of the formalism, but it rather points to a fundamental physical change. Of all unsolved problems of QFT, the ones hidden behind the infrared divergencies caused by massless vector potentials have remained the hardest. Though particle physicists have use recipes as e.g. photon-inclusive cross sections in QED and string-bridged quark-antiquark pairs in order to describe hadronic jets, there has been no spacetime understanding of these phenomena.

The message offered by the new SLF setting is that one should not address problems of perturbative interacting zero mass vector mesons directly, but rather treat them as limiting cases of their massive counterpart. The first step consists in computing the correlations functions in the massive theory. The Hilbert space positivity remains encoded in the Wightman positivity of the vacuum expectation values in the massless limit [30]. The massless operator theory and its Hilbert space can be reconstructed from the massless limit of these expectation values; in this way one avoids the futile attempt of trying to understand its Hilbert space in terms of particle concepts of the massive theory.

Such problems are outside the conceptual range of the BRST gauge formalism; the locality of fields in Krein space is not that of the physical Einstein causality even if it looks the same. In contrast to the Hilbert space setting which, as emphasized before, signals the problems of zero mass limits already in first order perturbation theory, gauge theory masks them. The BRST formalism continues to work in the massless limit, but its physical range is limited to gauge-invariant local observables and prescriptions for photon-inclusive cross sections (whose gauge invariance is not obvious). The success of the gauge-theoretical description of the Standard Model is based on the perturbative construction of the $S$-matrix for interactions of massive vector mesons; a deeper understanding of the physical content hidden behind infrared divergen- cies requires the string-local setting; here the Hilbert space positivity (off-shell unitarity) is indispensable.

In the present work the calculations within the SLF Hilbert space setting were limited to the on-shell $S$-matrix. The important task of extending the SLF setting to the construction of interacting string-local fields (off-shell unitarity) will be addressed in future publications. The remainder of this concluding section present an outlook about what one may expect from such an extension for a better understanding of the infrared problems.

The present picture based on perturbative calculations in gauge theory is that, although the perturbative scattering amplitudes are logarithmically infrared-divergent, the photon-inclusive cross section remains finite and gauge invariant [33]. It is believed that the logarithmic on-shell divergencies for scattering of charge-carrying particles result from a perturbative expansion of the "infraparticle structure". The latter is based on the idea that the interaction with the infrared photons "dissolves" the mass-shell of charged particles by converting the mass-shell poles into a milder cut-like singularity with a coupling-dependent power behavior. Such a "softened mass-shell singularity" would be too weak to counteract the dissipation of wave packets in the large time limits in LSZ scattering theory which then accounts for the vanishing of the scattering amplitude. The logarithmic divergencies of the perturbative scattering amplitude result from the perturbative expansion of the softened mass-shell.

In order to reconcile the vanishing scattering amplitudes with the photon-inclusive cross section one again replaces the photons by massive vector mesons of a small mass $m$. The number $N(m)$ of contributing vector mesons below a given invariant energy increases with $m \rightarrow 0$, but the inclusive cross section remains nontrivial while the individual amplitudes approach zero [33]. In the SLF setting all objects which enter this argument are physical.

The change of the mass-shell singularity in the infraparticle picture is supported by soluble two-dimensional models, of which the simplest was already constructed in the 1960s [34]. The solution $\psi(x)=\psi_{0}(x) \exp i g \varphi$ of the twodimensional derivative coupling $g \bar{\psi} \gamma_{\mu} \psi \partial^{\mu} \varphi$ provides an example in which the mass-shell of $\psi_{0}(x)$ changes into a $g$-dependent power singularity in the massless limit of the scalar field $\varphi$. The perturbative expansion of the exponential function before taking the massless limit leads to the logarithmic infrared divergencies. The simplicity of the model permits to study infrared behavior without dynamical complications (see the remarks at the end of Sect. 3).

The SLF Hilbert space setting is expected to lead to an understanding of infrared phenomena in terms of largedistance properties of string-local charge-carrying fields. For this one would have to extend the $S$-matrix formalism to correlation functions of string-local fields and define the massless theory in terms of the massless limit of correspond- 
ing massive correlation. The important role of the differential calculus on de Sitter space of space-like directions is to enable the implementation of the independence on inner string directions. In the case of the $S$-matrix all $e^{\prime} s$ are inner, whereas in the extension to vacuum expectation values one has to distinguish between the string directions of those fields whose correlation functions one wants to calculate and the inner $e^{\prime} s$ of the perturbative internal propagators.

This is reminiscent of gauge invariance, except that the BRST $\mathfrak{s}$-operation has no connection with spacetime and cannot distinguish between "inner and outer" gauge invariance. Without the local nature of the $d$ calculus, in which the $d_{e}$ differentials acts on individual fluctuating $e$ directions, it would not be possible to differentiate between outer and inner directional fluctuations. Needless to add that string-localization bears no relation to String Theory which, despite its name and in spite of many attempts of its defenders to connect it to QCD strings, bears no relation to localization in spacetime.

There is an important structural difference between interacting strings in the presence of mass gaps and massless strings. Whereas in the former case the correlation functions of string-local fields decrease exponentially for large spacelike separations (linked cluster property) [21] and the string directions can be freely changed by Lorentz transformation, the strings of charge-carrying particles in QED are "rigid". This leads to the spontaneously breaking of Lorentz invariance in charged sectors [28]. Needless to add that the correct QFT analog of long-range interactions (Coulomb potentials) in quantum mechanics are string-local charge-carrying fields in the Hilbert space description of QED.

A more radical change is expected to occur in the massless limit of massive self-interacting vector mesons. As explained before, the logarithmic divergent perturbative scattering amplitudes of charge-carrying particles are viewed as resulting from an illegitimate interchange of the limit $m \rightarrow 0$ with the perturbative expansion in a situation in which the non-perturbative limit vanishes. This suggests to view logarithmic divergencies in off-shell correlation functions of QCD containing gluon and quark fields as signaling confinement. In this case the only surviving vacuum expectation values would be those of point-local composites (gluonium, hadrons) and string-bridged compact localized $q \bar{q}$ pairs. A situation in which the basic fields (in terms of which one defines interactions) vanish cannot be directly described within the known formulation of QFT; one needs to define such interactions as massless limits.

This picture receives additional support by noticing a significant structural difference between QED and QCD strings. String-local vector potentials in QED are line integrals over observable field strengths and hence can be viewed as global limits of local observables. But interacting string-local fields in QCD cannot be represented in this way; their localization is inherently non-compact and their appearance in correlation functions would cause problems with causality. Confinement in the sense of vanishing of all vacuum expectation values containing such inherent non-compact strings avoids such causality problems.

It has been shown that perturbative $Q C D$ correlation functions in covariant gauges remain finite [35]. But the gauge which formally corresponds to the string-local Hilbert space formulation is the non-covariant axial gauge. It was abandoned a long time ago because it leads to uncontrollable (ultraviolet mixed with infrared) divergencies.

But what seems to be a curse in gauge theory turns out to be a blessing in the SLF Hilbert space setting. The latter turns the ill-defined noncovariant axial gauge parameter in Krein space into fluctuating directions of covariant string-local fields which act in Hilbert space. In this way the global on-shell unitarity of gauge theory is extended to local correlation functions of fields. This extension is of special importance in zero mass limits when the field-particle relation is lost. The SLF Hilbert space setting permits to address structural changes in massless limits which remain outside the reach of gauge theory, as they occur in the infraparticle structure of QED and the expected QCD confinement.

To achieve this one must extend the SLF of the present work to the perturbative calculation of correlation functions for massive string-local fields. Only in this way will one be able to understand phenomena as confinement in which the fundamental fields disappear in the massless limit and only their imprint on their composites (e.g. hadrons, and gluonium) and the disintegration of string-bridged $q \bar{q}$ pairs remains. Our present understanding of QFT permits no description of fields which, although not present in the formalism, yet assert their presence in "what they leave behind". The only known way to deal with such a situation is to view it as the massless limit of a massive model in a (necessarily string-local) Hilbert space setting.

Acknowledgments The interest in the construction of string-local fields and the study of their properties dates back to the 2005 joint work with Jens Mund and Jakob Yngvason. Afterwords Jens and I became interested in the use of these ideas for a reformulation of gauge theory. Whereas he started to look into the more ambitious project to extend the causal Epstein-Glaser setting from point-local to string-local fields, my interest was to relate the new ideas with the old ideas around the Schwinger-Swieca charge screening with which I had some familiarity from my collaboration with Swieca during the 1970s. So Jens and I decided to temporarily follow these interests separately in order to afterwards continue the shared project. I am deeply indebted to him for many discussions and for making his manuscript available to me. I am also indebted to José M. Gracia-Bondia for helpful comments. Last but not least, I thank Raymond Stora for his critical sympathy and helpful suggestions with which he accompanied the development of this project for more than a year.

Open Access This article is distributed under the terms of the Creative Commons Attribution 4.0 International License (http://creativecomm ons.org/licenses/by/4.0/), which permits unrestricted use, distribution, and reproduction in any medium, provided you give appropriate credit 
to the original author(s) and the source, provide a link to the Creative Commons license, and indicate if changes were made.

Funded by SCOAP ${ }^{3}$.

\section{References}

1. J. Schwinger, Trieste Lectures, 1962 (IAEA, Vienna, 1963)

2. J.A. Swieca, Phys. Rev. D 13, 312 (1976)

3. B. Schroer, A. Jorge, Swieca's contributions to quantum field theory in the $60 \mathrm{~s}$ and 70 s and their relevance in present research. Eur. Phys. J. H 35, 53-88 (2010)

4. G. Scharf, Quantum Gauge Theory, A True Ghost Story (Wiley, New York, 2001)

5. A. Aste, G. Scharf, M. Duetsch, J. Phys. A 30, 5785 (1997)

6. M. Duetsch, J.M. Gracia-Bondia, F. Scheck, J.C. Varilly, Quantum gauge models without classical Higgs mechanism. Eur. Phys. J. C 89, 599 (2012). arXiv:1001.0932

7. S. Mandelstam, Ann. Phys. 19, 1 (1962)

8. B.S. De Witt, Phys. Rev. 125, 2189 (1962)

9. D. Buchholz, Commun. Math. Phys. 85, 40 (1982)

10. J. Yngvason, Zero-mass infinite spin representations of the Poincaré group and quantum field theory. Commun. Math. Phys. 18, 195 (1970)

11. R. Brunetti, D. Guido, R. Longo, Modular localization and Wigner particles. Rev. Math. Phys. 14, 759 (2002)

12. B. Schroer, Modular localization and the holistic structure of causal quantum theory, a historical perspective. SHPMP 49, 109 (2015)

13. B. Schroer, The Einstein-Jordan conundrum and its relation to the ongoing foundational research in local quantum physics. Eur. Phys. J. H 38, 13 (2013)

14. J. Mund, B. Schroer, J. Yngvason, String-localized quantum fields and modular localization. CMP 268, 621 (2006). arXiv:math-ph/0511042

15. R. Longo, V. Morinelli, K.-H. Rehren, Where infinite spin particles are localizable. arXiv: 1505.01759

16. J. Mund, Progr. Math. 251, 199 (2007)
17. J. Mund, String-localized quantum fields, modular localization, and gauge theories, in New Trends in Mathematical Physics, Selected contributions of the XVth International Congress on Mathematical Physics ed. by V. Sidoravicius (Springer, Dordrecht, 2009), p. 495

18. B. Schroer, An alternative to the gauge theory setting. Found. Phys. 41, 1543 (2011). arXiv:1012.0013

19. B. Schroer, A Hilbert space setting for interacting higher spin fields and the Higgs issue. Found. Phys. 45, 219 (2015). arXiv: 1407.0360

20. B. Schroer, The ongoing impact of modular localization on particle theory. SIGMA 10, 085 (2014)

21. D. Buchholz, K. Fredenhagen, Commun. Math. Phys. 84, 1 (1982)

22. R. Haag, Local Quantum Physics (Springer, Heidelberg, 1996)

23. D. Buchholz, K. Fredenhagen, Nucl. Phys. B 154, 226 (1979)

24. J. Mund, Modular localization of massive particles with "any" spin in $\mathrm{d}=2+1$. J. Math. Phys. 44, 2037 (2003)

25. P. Leyland, J. Roberts, D. Testard, Duality for Quantum Free Fields (Centre de Physique Théorique, CNRS, Marseille, 1978)

26. K.-H. Rehren, Local quantum observables in the anti-deSitterconformal QFT correspondence. Phys. Lett. B 493, 383 (2000). arXiv:hep-th/0003120

27. M. Duetsch, K.-H. Rehren, Generalized free fields and the AdS-CFT correspondence. Ann. Henri Poincare 4, 613 (2003). arXiv:math-ph/0209035

28. J. Fröhlich, G. Morchio, F. Strocchi, Phys. Lett. 89B, 61 (1979)

29. M. Duetsch, B. Schroer, J. Phys. A 33, 4317 (2000)

30. R.S. Streater, A.S. Wightman, PCT, Spin and Statistics and All That (Benjamin, New York, 1964)

31. H. Ezawa, J.A. Swieca, Commun. Math. Phys. 5, 330 (1967)

32. D. Buchholz, J. Roberts, New light on infrared problems: sectors, statistics, symmetries and spectrum. arXiv:1304.2794

33. D. Yenni, S. Frautschi, H. Suura, Ann. Phys. 13, 370 (1961)

34. B. Schroer, Infrateilchen in der Quantenfeldtheorie. Fortschr. Phys 173, 1527 (1963)

35. S. Hollands, Renormalized quantum Yang-Mills fields in curved spacetime. Rev. Math. Phys. 20, 1033 (2008). arXiv:0705.3340 